\section{Explain Yourself}

Statistically speaking, air travel is very safe with respect to major hazards, but there is still a very high risk of being assigned a seat next to a garrulous, usually aged person. Why is seating preference restricted to "aisle" or "window?" Why can't there be a "no conversation" section? There are few effective defenses against itinerant conversationalists, but only the most insistent of the breed will interrupt your reading, so it is a good idea to have a very thick novel to read, though you risk being asked for a review or even a book-club-style discussion if the fellow traveler happens to have read the book, so it is best to have obscure tastes in literature. Safer yet, just dig into a sheaf of manuscripts or proposals that you have to review. I have also begun to use a nice pair of noisecanceling headphones for in-flight entertainment or music stored on my laptop. As they are a usefully visible discouragement to the chatty traveler, I often keep them on even when I am not listening to anything.

Eventually, however, you will be drawn into conversation. The conventions of polite airplane conversation allow your fellow traveler to ask where you are going (same place as YOU, of course!) and why. Most of our travel is work-related, so this inevitably leads to the question of what you do. I know that some of you pretend to be Ford dealers, management consultants, or tax inspectors in order to avoid explaining what a materials scientist is, so let's try to find a better way to explain ourselves.

We often describe our subject in terms of structure, properties, and processing, possibly throwing in "performance" to create a tetrahedral model that is instantly appealing to those of us who deal with atomic models in our daily labor. Of course, the rest of the world has not a clue what this means, and they are not even particularly attracted to ball-and-stick models of anything at all, let alone atomic metaphors for intellectual activities. We have to recognize that the description of which we are all so fond is only a good "internal" description for explaining our field to those who are already in it.

What we need is an "external" description that allows a lay person to believe that she/he understands it. It is always a good idea to start with something that your interlocutors understand, or at least think they do, and following Einstein's dictum, the explanation should be as simple as possible, but no simpler.

Let's start with the technical details, for our own benefit. The figure shows a sort of Venn diagram of the domains of physics, chemistry, biology, and materials that is at least reasonably accurate. There is some overlap of physics and chemistry, representing chemical physics, or physical chemistry, depending on which side you come from. Similar overlaps exist between biology and the other two "basic sciences," and there is even a region where all three overlap. A large part of physics falls within the domain of materials, as does a large part of chemistry and a small (but definitely growing) part of biology. Almost all of the overlaps between the basic sciences fall inside the domain of materials, but there is also a large part of materials that is neither physics, nor chemistry, nor biology. This part has more to do with materials engi-

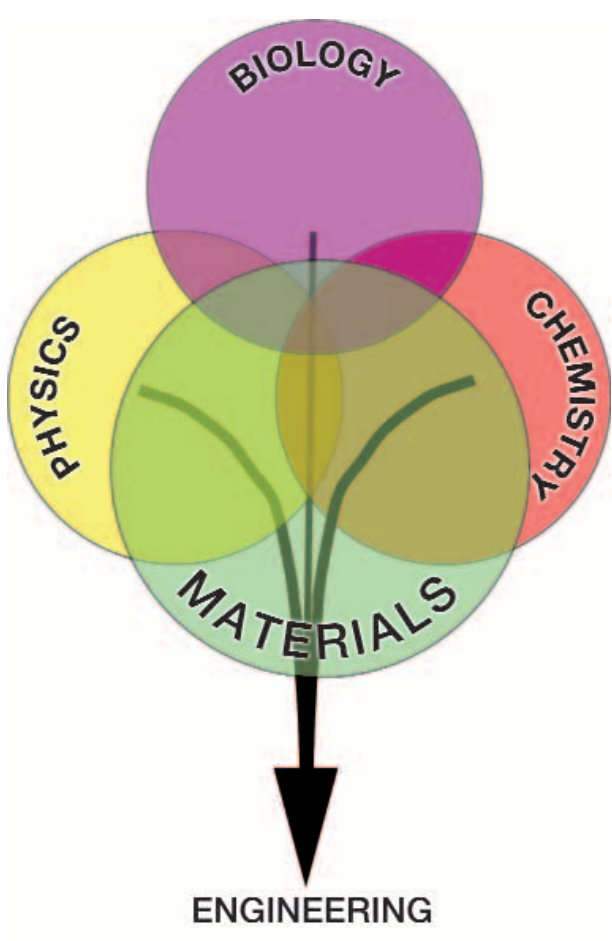

Figure. What do you see in this image? neering than materials science.

Now think about what YOU are working on. The chances are that you are struggling with ways to make some fundamental discovery in physics or chemistry into a usable product designed (or about to be designed) by a mechanical engineer or an electrical engineer. When your work is done, hopefully the next stage will be turned over to a manufacturing engineer who will figure out how to make the device, whatever it is, reproducibly and inexpensively. The arrow in my diagram shows (in grossly simplified form) the flow of knowledge that is required to make sophisticated products available to consumers. So here is the short definition of our field: Materials science and engineering forms the exclusive knowledge pathway through which fundamental discoveries are turned into useful products. I can think of no example in modern technology in which there has not been a role of this kind for materials science.

Most members of the public seem to think that they know what physicists and chemists do because they have had some elementary introduction to these subjects in school, but they haven't a clue about structure, processing, and properties. My little diagram seems to be reasonably well understood, and it is easily reproduced on an airline paper napkin (though some of those could use some improvement in their properties). The most important thing, from a PR perspective, is to remember that the circle representing materials always has to be larger than the other three!

Now, you need to be aware that a diagram like this one brings forth reactions distinctive to different groups of people. The very young are apt to see a bunch of balloons in this picture, which is OK as long as they associate materials with fun. Middle-aged men, I have found, react by saying something like "then this materials thing is like an enlarged prostate, limiting the flow?" But some women, especially those of childbearing age, see the materials domain as the womb in which new technologies are nurtured to the point of independent survivability. I rather like the women's viewpoint here: Who wouldn't want to work in a field that considers itself to be the "womb of technology?"

ALEX KING 